\title{
Binet Stage A
}

National Cancer Institute

\section{Source}

National Cancer Institute. Binet Stage A. NCI Thesaurus. Code C80134.

Patients have no anemia or thrombocytopenia and fewer than three areas of lymphoid involvement. 